\title{
Research on active compounds regarding SGMD for the treatment of COVID-19 based on network pharmacology and molecular docking
}

Ma Donglai ( $\square$ mdl_hebei@aliyun.com )

Hebei University of Chinese Medicine

Yuxin Jia

Hebei University of Chinese Medicine

Mingdong Si

Hebei University of Chinese Medicine

Huigai Sun

Hebei University of Chinese Medicine

Huiru Du ( $\nabla 73325390 @ Q Q . c o m$ )

Hebei University of Chinese Medicine

Jing Li ( $\nabla$ wy1971401@163.com )

Hebei University of Chinese Medicine

\section{Research}

Keywords: Six Gentlemen Decoction (SGD), Flavonoids, COVID-19, Network pharmacology, Molecular docking

Posted Date: August 19th, 2020

DOl: https://doi.org/10.21203/rs.3.rs-60439/v1

License: (9) This work is licensed under a Creative Commons Attribution 4.0 International License. Read Full License 


\section{Abstract}

Background: Retrieve Curative effect of Six Gentlemen Modified Decoction (SGMD) in treating with coronavirus disease ( COVID-19) by network pharmacology and verify its authenticity by molecular docking.

Methods: The chemical constituents, effective components, and action targets were screened using TCMSP. COVID-19 related targets were retrieved by the GeneCards and NCBI databases, and drug targets and disease targets were mapped by Venny to obtain potential targets for treatment. The regulatory network of traditional Chinese medicine (TCM) compounds was established with Cytoscape to obtain the key components, and the PPI network and its network topology were established with the Bisogenet and CytoNCA plug-ins to obtain the core targets. Bioconductor was used for GO function analysis and KEGG pathway analysis to obtain the relevant functions and pathways.

Results: 173 effective components, 253 targets, and 348 targets related to COVID-19 were obtained after screening, 50 cross targets were shown, and the key components of the top 15 are flavonoids such as quercetin, luteolin, kaempferol, naringenin, licochalcone A, etc. The top 28 core targets include TP53, EGFR, SRC, AR, ABL1, and others. Biological processes such as the responses to metal ions, molecules of bacterial origin, lipopolysaccharide, toxic substances, and oxidative stress were involved. The main pathway involved the AGE-RAGE signaling pathway in diabetic complications as well as the TNF and IL17 signaling pathways. The average binding energies of the first three core components connected with $6 \mathrm{LU7}$ and $1 \mathrm{R} 42$ were $-4.16 \mathrm{~kJ} / \mathrm{mol}$ and $-4.12 \mathrm{~kJ} / \mathrm{mol}$, respectively.

Conclusion: The core compounds of SGMD can spontaneously combine with SARS-CoV-2 3CL hydrolase and ACE2 to treat COVID-19.

\section{Background}

Coronavirus disease is an epidemic disease with a long incubation period, strong infectivity and fast transmission rate [1] that has been declared a public health emergency by the world health organization. This kind of disease spreads quickly, easily infecting all kinds of people. It can be transmitted via droplets, aerosols, close contact, or the fecal-oral route. The pathogenesis has not been studied, so there is no specific medicine for treatment. Based on syndrome differentiation, TCM has played its unique advantages in the treatment of diseases since ancient times. Currently, traditional Chinese medicine has the advantages of treating both symptoms and root causes as well as being safe and reliable and exhibiting significant curative effects and less side effects in the treatment of COVID-19.

The national health commission issued the diagnosis and treatment protocol for COVID-19 trial version 7 (On March 4, 2020) [2]. This scheme pointed out that Chinese medicine used SGMD to control the type of lung and spleen deficiency syndrome of convalescent patients. The composition of this decoction includes $9 \mathrm{~g}$ of Pinelliae Rhizoma, $10 \mathrm{~g}$ of Citri Reticulatae Pericarpium, $15 \mathrm{~g}$ of Codonopsis Radix, $30 \mathrm{~g}$ of Astragali Radix, $10 \mathrm{~g}$ of Atractylodis Macrocephalae Rhizoma, $15 \mathrm{~g}$ of Poria, $10 \mathrm{~g}$ of Pogostemonis Herba, 
$6 \mathrm{~g}$ of Amomi Fructus and $6 \mathrm{~g}$ of Glycyrrhizae Radix et Rhizoma. SGD was recorded in the book entitled, "Ancient and Modern Famous Medical Prescriptions". The decoction strengthens the spleen, supplements the lung, and benefits the qi for resolving dampness. It is often used for the treatment of spleen stomach qi deficiency and retention of dampness and stagnation of qi. Astragali Radix and Pogostemonis Herba were added into this formula. Astragali Radix is the essential medicine for elevating yangqi. It can tonify the qi of lung and spleen as well as cure internal heat and diabetes. Pogostemonis Herba is a kind of aromatic damp-resolving drug, and it is recorded in the book "Medicinal meaning". It is used along with fragrant qi, which is good for regulating the spleen and stomach of the middle jiao, and it has the effect of activating the spleen and enhancing appetite. It can also reduce distension syndrome of the middle jiao and then cure vomiti and cholera. Modern studies [3-6] have shown that the decoction warms the spleen and stomach, invigorating and regulating qi. Thus, it can be used to clear the qi of the middle jiao, regulate the gastrointestinal tract, enhance the appetite of patients, and regulate metabolism, endocrine and immune functions.

This study is based on network pharmacology and sought to screen the active ingredients of SGMD in order to determine its effective components and targets. The COVID-19 screening found the intersection of drug targets and disease targets. Furthermore, the mechanism of action of this prescription was elucidated to provide a scientific basis for the development and clinical application of COVID-19 drugs.

\section{Materials And Methods}

\subsection{Retrieval of chemical compositions}

Based on the TCMSP database (http://lsp.nwu.edu.cn /tcmsp.php), the key words of "Pinelliae Rhizoma", "Citri Reticulatae Pericarpium", "Codonopsis Radix", " Astragali Radix", "Atractylodis Macrocephalae Rhizoma", "Poria", "Pogostemonis Herba", "Amomi Fructus" and "Glycyrrhizae Radix et Rhizoma" were searched to obtain the chemical compositions and targets of the drug.

\subsection{Retrieval of effective ingredients and targets}

In the above retrieval results, the bioavailability $(\mathrm{OB})>30 \%$ and drug-like property $(\mathrm{DL})>0.18$ were selected as the screening conditions for the secondary retrieval to collect the effective ingredients and targets of various herbs in this prescription, and the collected target proteins were inputted into the UniProt database (https://www.uniprot.org/) for gene name correction.

\subsection{Retrieval of COVID-19 related targets}

The COVID-19-related targets were identified in the GeneCards (http://www.genecards.org/) and NCBI (https://www.ncbi.nlm.nih.gov/) databases, respectively, using the phrase "novel coronavirus" as a keyword.

2.4 Prediction of potential targets of COVID-19 in drug therapy 
The intersection of drug targets and disease targets was obtained using the Venny mapping software, and the cross targets were the potential targets of SGMD relevant to COVID-19.

\subsection{The establishment of regulatory network of TCM compounds}

The targets of SGMD relevant to the treatment of COVID-19 were uploaded to the Cytoscape software 3.7.1 区http://www. cytoscape. Org/). Then, we obtained an interaction diagram of the TCM - component target - disease network, which means the regulation network of TCM compounds was constructed.

\subsection{The establishment of an PPI network}

The names of the cross targets were imported into the Bisogenet plug-in in cytoscape-3.7.1 software to create a new network, and the species option was selected as "Homo sapiens". Then, the construct protein interaction network button was selected. At last, we generated the PPI network, which was used to identify the functional partnerships and interactions between proteins.

\subsection{Network topology analysis}

Traditional Chinese medicine, effective ingredients, targets and disease are the nodes in the network of TCM compounds regulation. When there is an association among these nodes, the Networkanalysis plugin in cytoscape-3.7.1 software can be used to analyze the topological structure. In addition, the core targets of SGMD relevant to COVID-19 can be screened out.

\subsection{GO function analysis and KEGG pathway analysis}

The Bioconductor database (http://www.bioconductor.org/) was used for the GO function analysis and the enrichment of KEGG. The targets of drugs treatment related to COVID-19 were entered, and the column diagram of the molecular function, cell composition, biological process and pathway process were determined. Finally, we speculated the most probable mechanism of action in the treatment of COVID-19.

\subsection{Components-targets molecular docking}

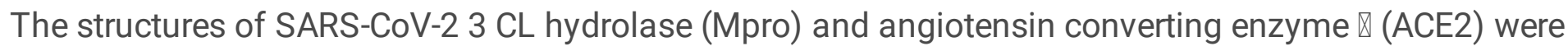
found using the RCSB database (http://www.rcsb.org), and the result showed that the protein codes (PDB IDS) were 6 lu7 and $1 \mathrm{r} 42$, respectively. Mgl tools 1.5 .6 (ttp://mgltools.scripps.edu/) were used for protein processing, and the results were saved in the PDBQT format after water removal and hydro-charging. The ligands (active compounds) in the form of Mol2 were treated in the same way and stored in the same format for later use. The dock key components and the above two targets were used with AutoDock 4.2.6 (http://autodock.scripps.edu/).Then,the docking files were converted to PDB format with OpenBabel2.4.1 software (http://openbabel.org/wiki/Main_Page). Finally, the results of the docking between the components and the targets were observed and analyzed in Pymol 2.3.3 (hts:// pymol.org/2/). 


\section{Results}

\subsection{Retrieval of chemical compositions, effective components and targets}

There were a large number of pharmaceutical chemical components in SGMD that were retrieved by TCMSP. A total of 194 effective ingredients were obtained after the second retrieval based on the screening conditions of bioavailability $(\mathrm{OB})>30 \%$ and drug-like property $(\mathrm{DL})>0.18$.Among them, 13 in Pinelliae Rhizoma, 5 in Citri Reticulatae Pericarpium, 21 in Codonopsis Radix, 20 in Astragali Radix, 7 in Atractylodis Macrocephalae Rhizoma, 15 in Poria, 11 in Pogostemonis Herba, 10 in Amomi Fructus and 92 in Glycyrrhizae Radix et Rhizoma were found. Due to the multi-component and multi-target characteristics of traditional Chinese medicine, there exists the phenomenon that multiple traditional Chinese medicines contain the same component. 173 effective ingredients and 253 corresponding action targets were obtained after removing duplicates. The basic information of some of the effective ingredients of SGMD is shown in table 1.

Table 1 Basic information of active ingredients of SGMD 


\begin{tabular}{|c|c|c|c|c|}
\hline Herbs & MolID & MolName & OB & DL \\
\hline \multirow{3}{*}{$\begin{array}{l}\text { Atractylodis } \\
\text { Macrocephalae } \\
\text { Rhizoma }\end{array}$} & MOL000020 & 12-senecioyl-2E,8E,10E-atractylentriol & 62.4 & 0.22 \\
\hline & MOL000021 & $\begin{array}{l}\text { 14-acetyl-12-senecioyl-2E,8E,10E- } \\
\text { atractylentriol }\end{array}$ & 60.31 & 0.31 \\
\hline & MOL000022 & $\begin{array}{l}\text { 14-acetyl-12-senecioyl-2E,8Z,10E- } \\
\text { atractylentriol }\end{array}$ & 63.37 & 0.3 \\
\hline \multirow[t]{6}{*}{ Pinelliae Rhizoma } & MOL001755 & 24-Ethylcholest-4-en-3-one & 36.08 & 0.76 \\
\hline & MOL002670 & Cavidine & 35.64 & 0.81 \\
\hline & MOL002714 & baicalein & 33.52 & 0.21 \\
\hline & MOL002776 & Baicalin & 40.12 & 0.75 \\
\hline & MOL000358 & beta-sitosterol & 36.91 & 0.75 \\
\hline & MOL000449 & Stigmasterol & 43.83 & 0.76 \\
\hline \multirow[t]{3}{*}{$\begin{array}{l}\text { Citri Reticulatae } \\
\text { Pericarpium }\end{array}$} & MOL005100 & $\begin{array}{l}\text { 5,7-dihydroxy-2-(3-hydroxy-4- } \\
\text { methoxyphenyl) chroman-4-one }\end{array}$ & 47.74 & 0.27 \\
\hline & MOL005815 & Citromitin & 86.9 & 0.51 \\
\hline & MOL005828 & nobiletin & 61.67 & 0.52 \\
\hline \multirow[t]{5}{*}{ Codonopsis Radix } & MOL002140 & Perlolyrine & 65.95 & 0.27 \\
\hline & MOL005321 & Frutinone A & 65.9 & 0.34 \\
\hline & MOL000006 & luteolin & 36.16 & 0.25 \\
\hline & MOL007059 & 3-beta-Hydroxymethyllenetanshiquinone & 32.16 & 0.41 \\
\hline & MOL008400 & glycitein & 50.48 & 0.24 \\
\hline Poria & MOL000296 & hederagenin & 36.91 & 0.75 \\
\hline \multirow[t]{5}{*}{ Pogostemonis Herba } & MOL005573 & Genkwanin & 37.13 & 0.24 \\
\hline & MOL005911 & 5-Hydroxy-7,4'-dimethoxyflavanon & 51.54 & 0.27 \\
\hline & MOL005916 & irisolidone & 37.78 & 0.3 \\
\hline & MOL005918 & phenanthrone & 38.7 & 0.33 \\
\hline & MOL005921 & quercetin 7-0- $\beta$-D-glucoside & 49.57 & 0.27 \\
\hline \multirow[t]{3}{*}{ Astragali Radix } & MOL000239 & Jaranol & 50.83 & 0.29 \\
\hline & MOL000354 & isorhamnetin & 49.6 & 0.31 \\
\hline & MOL000371 & 3,9-di-0-methyInissolin & 53.74 & 0.48 \\
\hline
\end{tabular}




\begin{tabular}{|c|c|c|c|c|}
\hline & MOL000378 & 7-0-methylisomucronulatol & 74.69 & 0.3 \\
\hline & MOL000379 & $\begin{array}{l}\text { 9,10-dimethoxypterocarpan-3-O-B-D- } \\
\text { glucoside }\end{array}$ & 36.74 & 0.92 \\
\hline & MOL000380 & $\begin{array}{l}\text { (6aR,11aR)-9,10-dimethoxy-6a,11a-dihydro- } \\
\text { 6H-benzofurano [3,2-c] chromen-3-ol }\end{array}$ & 64.26 & 0.42 \\
\hline & MOL000387 & Bifendate & 31.1 & 0.67 \\
\hline & MOL000392 & formononetin & 69.67 & 0.21 \\
\hline & MOL000417 & Calycosin & 47.75 & 0.24 \\
\hline & MOL000422 & kaempferol & 41.88 & 0.24 \\
\hline & MOL000442 & 1,7-Dihydroxy-3,9-dimethoxy pterocarpene & 39.05 & 0.48 \\
\hline \multirow[t]{2}{*}{ Amomi Fructus } & MOL001755 & 24-Ethylcholest-4-en-3-one & 36.08 & 0.76 \\
\hline & MOL001771 & poriferast-5-en-3beta-ol & 36.91 & 0.75 \\
\hline \multirow[t]{14}{*}{$\begin{array}{l}\text { Glycyrrhizae Radix et } \\
\text { Rhizoma }\end{array}$} & MOL004941 & $\begin{array}{l}\text { (2R)-7-hydroxy-2-(4-hydroxyphenyl) } \\
\text { chroman-4-one }\end{array}$ & 71.12 & 0.18 \\
\hline & MOL001792 & DFV & 32.76 & 0.18 \\
\hline & MOL004835 & Glypallichalcone & 61.6 & 0.19 \\
\hline & MOL004841 & Licochalcone B & 76.76 & 0.19 \\
\hline & MOL004985 & icos-5-enoic acid & 30.7 & 0.2 \\
\hline & MOL004996 & gadelaidic acid & 30.7 & 0.2 \\
\hline & MOL003896 & 7-Methoxy-2-methyl isoflavone & 42.56 & 0.2 \\
\hline & MOL000500 & Vestitol & 74.66 & 0.21 \\
\hline & MOL004957 & HMO & 38.37 & 0.21 \\
\hline & MOL004328 & naringenin & 59.29 & 0.21 \\
\hline & MOL000392 & formononetin & 69.67 & 0.21 \\
\hline & MOL000422 & kaempferol & 41.88 & 0.24 \\
\hline & MOL000417 & Calycosin & 47.75 & 0.24 \\
\hline & MOL000098 & quercetin & 46.43 & 0.28 \\
\hline
\end{tabular}

\subsection{Retrieval of COVID-19 related targets}

The GeneCards and NCBI databases were used to retrieve the keyword "novel coronavirus". In total, 346 related targets were found in the GeneCards database, and 48 related targets were found in the NCBI 
database, among which 46 were common targets. After removing common targets, 348 targets related to COVID-19 were obtained.

\subsection{Potential targets of COVID-19 in drug therapy}

The Venny mapping software was used to perform intersection alignment of 253 effective ingredients regulated targets retrieved from the TCMSP database, and a total of 348 targets related to COVID-19 identified from the GeneCards and NCBI databases. 50 cross-targets were obtained, which were potential targets for the treatment of COVID-19 by SGMD, as shown in fig. 1 .

\subsection{Analysis of regulatory network of TCM compounds}

After the cross-targets were imported into Cytoscape 3.7.1 software (http://www. cytoscape.org/), the TCM compound regulatory network was obtained, with 168 nodes and 588 edges, as shown in fig. 2 . The 118 effective ingredients in SGMD acted on 50 cross-targets of drugs and diseases to treat COVID-19. In fig. 2, the ginger yellow nodes represent the effective ingredients of Pinelliae Rhizoma, the grey nodes represent the effective ingredients of Citri Reticulatae Pericarpium, and the purple nodes represent the effective ingredients of Codonopsis Radix. The light-yellow nodes represent the effective ingredients of Astragali Radix, and the yellow node represents the effective ingredients of Atractylodis Macrocephalae Rhizoma, while the brown node represents the effective ingredients of Poria, and the pink node represents the effective ingredients of Pogostemonis Herba. The blue node represents the effective ingredients of Amomi Fructus, and the green node represents the effective ingredients of Glycyrrhizae Radix et Rhizoma, while the red nodes represent the chemical components that exist in multi-herb Chinese medicine and act on COVID-19. The sky-blue nodes represent the potential targets of drug treatment, and each edge represents the interaction between the effective ingredients and the potential therapeutic targets. There were 8 red nodes in total. MOL000239-Jaranol, MOL000354-isorhamnetin, MOL000392-formononetin, MOL000417-claycosin, and MOL000422-kaempferol were the common components of Astragali Radix and Glycyrrhizae Radix et Rhizoma; MOL004328- naringin was the common component of Citri Reticulatae Pericarpium and Glycyrrhizae Radix et Rhizoma; MOL003896-7-methoxy-2-methyl isoflavone was the common component of Codonopsis Radix and Glycyrrhizae Radix et Rhizoma; and MOL000098quercetin was the common component of Pogostemonis Herba, Astragali Radix and Glycyrrhizae Radix et Rhizoma. These common components are all flavonoids. The first 15 active ingredients, which were sorted in descending order of degree, were quercetin, luteolin, kaempferol, naringin, licochalcone A, nobiletin, irisolidone, baicalein, formononetin, isorhamnetin, 7-0-methylisomucronulatol, Odoratin, Vestitol, 7-Acetoxy-2-methylisoflavone and Quercetin der./3,3'-di-0-methylquercetin. These compounds are also flavonoids, which are key components in the treatment of COVID-19.

\subsection{PPI network}

Fifty drug and disease cross-targets were imported into the Bisogenet plug-in, and the protein-protein interaction (PPI) network was obtained after the network analysis, as shown in fig. 3 . A total of 2,133 target proteins were loaded. 


\subsection{Network topology analysis}

The CytoNCA plug-in was used to carry out the topological analysis of target proteins in the PPI network. In order of degree, 101 target proteins and sub-network 1 were obtained by filtering with DC>61 as the screening condition (fig. 4b). The resulting target proteins were filtered twice in betweenness order, and 28 target proteins and sub-network 2 were obtained by filtering with $B C>100$ as the screening condition (fig. 4c). The 28 targets were TP53, EGFR, SRC, AR, ABL1, GRB2, ABL1, GRB2, AKT1, MAPK1, HSP90AB1, BRCA1, CTNNB1, RAF1, MAP3K3, ESR1, SMAD2, HSP90AA1, YWHAZ, UBC, LYN, STAT3, HSPA8, PIK3R1, RB1, SMAD3, FYN, CASP3, IKBKG and SP1. As the core targets in the network, they are the most closely related to the treatment of COVID-19 by SGMD.

\subsection{GO function analysis and KEGG pathway analysis}

SGMD treatment targets related to COVID-19 were entered into Bioconductor for the GO functional analysis and KEGG enrichment analysis. $P$ value $<0.05$ was set as the default option, and the first 20 lines of the screening results were plotted into a histogram. The $\mathrm{GO}$ functional analysis includes the biological process (BP), molecular function (MF) and cell composition (CC). According to panels A, B and C in fig. 5, the relevant targets for the treatment of COVID-19 were mainly concentrated in biological processes such as responses to metal ions, molecules of bacterial origin, lipopolysaccharides, toxic substances and oxidative stress. The main molecular functions were concentrated in cytokine receptor binding, phosphatase binding, $\mathrm{BH}$ domain binding, cytokine activity and protein phosphatase binding. The main cellular components were concentrated in membrane raft, membrane microdomain and the membrane region. According to fig. $5 \mathrm{~d}$, the relevant targets for the treatment of COVID-19 were produced by treating the effect mainly through the AGE-RAGE signaling pathway in diabetic complications as well as the TNF and IL-17 signaling pathways.

\subsection{Target -KEGG pathway network}

Cytoscape 3.7.1 software was used to map the 20 pathways and path-related targets obtained from the KEGG enrichment analysis in order to understand the pathway mechanism. The hsa04933-AGE-RAGE signaling pathway in diabetic complications as well as the hsa04668-TNF and hsa04657-IL-17 signaling pathways are of great importance in the enriched pathways. MAPK1, MAPK3, RELA, IL6, MAPK14 and other targets also play important roles in this network (fig. 6).

\subsection{Molecular docking}

15 key components, including quercetin, luteolin, kaempferol, naringin, licochalcone A, nobiletin and irisolidone were connected with the SARS-CoV-2 3CL hydrolase and angiotensin converting enzyme 2 (ACE2) using the AutoDock 4.2.6 software. It is generally believed that the lower the binding energy of the ligand receptor, the easier the binding. According to the binding energy, the first three core components with the lowest molecular weight in connection with 6LU7 and 1R42 were identified as formononetin, Quercetin der., 7-Acetoxy-2-methylisoflavone and 7-Acetoxy-2-methylisoflavone, irisolidone and 
formononetin, respectively. Formononetin and 7-Acetoxy-2-methylisoflavone are bound by hydrogen bonds with SER-10, ALA-7, and LYS-5. Quercetin der. is bound by hydrogen bonding between SER-10, ALA7 and 6LU7. 7-Acetoxy-2-methylisoflavone is bound by hydrogen bonds formed between IL-256, MET-249 and 1R42, while irisolidone and formononetin is formed between SER-254 and ASN-250. The docking mode is shown in fig. 7.

\section{Discussion}

In the investigation and analysis of TCM symptoms of COVID-19 patients in convalescence, it was found that about $26.1 \%$ of the patients presented with deficiency of lung and spleen [7]. According to the diagnosis and treatment protocol for COVID-19 issued by the national health commission, the trial version 7 describes the symptoms of COVID-19 convalescent patients with deficiency of lung and spleen, and the main clinical manifestations are shortness of breath, fatigue and weakness, anorexia and nausea, fullness, and uncomfortable loose stool. The tongue is light and fat, and the coating is white and greasy. In traditional Chinese medicine, the spleen is the mother of the lung, and the lung is the main source of qi, while the spleen nourishes the qi. The lung is the master of qi, and the shortness of breath is the symptom of deficiency of lung qi. The spleen is the foundation of acquired constitution. Once the human body is in the state of spleen deficiency, production of qi and blood would be deficient, leading to water and body fluid metabolism disorders, which would then lead to the viscera loss of nutrition, water and grain transport disorders, internal generation of phlegm and dampness, which can cause the symptoms of fatigue, weakness, anorexia, nausea, fullness, and uncomfortable loose stool. Therefore, clinical treatment should follow the principle of strengthening the spleen, supplementing the lungs and benefiting the qi for resolving dampness. Multiple Chinese medicines of SGMD used in combination can restore the function of all vessels converging in the lung and promote digestion and absorption. In this study, network pharmacology and molecular docking technology were used to further explore the therapeutic mechanism of the recommended prescription of SGMD.

The network pharmacology results are as follows: there are 173 active ingredients and 253 targets that reflect the characteristics of multiple components and targets of traditional Chinese medicine. From the regulatory network of TCM compounds, the key components in the treatment of COVID-19 include flavonoids such as quercetin, luteolin, kaempferol, naringin, licochalcone A, nobiletin, irisolidone, baicalein, formononetin, isorhamnetin, 7-0-methylisomucronulatol, Odoratin, Vestitol, 7-Acetoxy-2methylisoflavone, Quercetin der., etc. The PPI network and its topology suggest that the core targets of this decoction are TP53, EGFR, SRC, AR, ABL1, GRB2, AKT1, MAPK1, and others, totalling 28 targets. The $\mathrm{GO}$ function analysis suggests that the biological processes of this decoction include responses to metal ions, molecules of bacterial origin, lipopolysaccharides, toxic substances and oxidative stress. The KEGG pathway analysis suggests that pathways play a therapeutic role of this decoction, including the AGE-RAGE signaling pathway in diabetic complications as well as the TNF and IL-17 signaling pathways. In addition, the targets with high frequencies involved in the above pathways are MAPK1, MAPK3, RELA, IL6, MAPK14. 
The molecular docking technique was used to verify the components and targets. The results were as follows: formononetin, Quercetin der. and 7-Acetoxy-2-methylisoflavone blocked the replication of the virus by binding to the 6 LU7 receptor protein through SER-10,ALA-7, LYS- 5 and other cytokines, and the binding energies were $-4.28,-4.12,-4.08$ (unit $\mathrm{kJ} / \mathrm{mol}$ ), with an average of $-4.16 .7-A c e t o x y-2-$ methylisoflavone, irisolidone and formononetin bind to the human receptor protein 1R42 to prevent virus invasion through the cytokines such as IL-256, MET-249, SER-254 and ASN-250, and the binding energies were $-4.26,-4.16$ and $-3.93 \mathrm{~kJ} / \mathrm{mol}$, with an average of $-4.12 \mathrm{~kJ} / \mathrm{mol}$. Previous studies have suggested that binding energies less than 0 indicate that the ligand and receptor can spontaneously bind [8]. The above results indicated that the core components of SGMD exhibited good binding properties with COVID-19related target proteins, which verified the therapeutic effect of this decoction.

Pharmacological studies have shown that formononetin may inhibit the inflammatory response of rats by controlling the expression of the Sph k1-s1p signaling pathway [9]. It can inhibit spleen cell apoptosis and improve its conversion rate, improve the innate immune function of mice [9], suppress the proliferation of leukemia HL-60 cells and increase the apoptosis rate [10] and protect myocardial cells [11]. Quercetin der. is a derivative of Quercetin. Quercetin can reduce the apoptosis rate of rat alveolar cells and inhibit inflammation by suppressing the expression of TGF- 31 , TNF- $a$ and so on [12]. In addition, studies have shown that quercetin also has a great diversity of pharmacological activities like antioxidation [13-15], antitumor [16-19], antiviral [20-23] and immunoregulation [24-26]. The pharmacological effects of irisolidone include anti-inflammatory, antioxidation, antibacterial and antitumor impacts [27]. MAPK1, MAPK3 and MAPK14 are all belong to the mitogen-activated protein kinase (MAPK). They are mainly engaged in cell activation, growth, proliferation, apoptosis and immunoregulation, which can inhibit the expression of inflammatory factors and reduce lung injury. RELA can promote the apoptosis of neutrophils [28] and reduce the inflammatory response. IL 6 is an inflammatory mediator, which is not only related to the occurrence and spread of inflammation [29-31], but it is also involved in the suppression of inflammation [32-34]. The AGE-RAGE signaling pathway reacts on cell activation and tissue damage and has the activity of promoting the expression and release of inflammatory factors [35]. The TNF and IL-17 signaling pathways also play a crucial part in the occurrence and development of inflammation [26, 36, 37].

\section{Conclusions}

In summary, through network pharmacology, this paper collated the effective ingredients, action targets, potential action targets, key components and core targets related to treating COVID-19 as well as the biological functions and pathways of potential action targets in SGMD. The molecular docking technology was used to verify the reliability of the therapeutic effect of this decoction.

This study is only based on the pharmacology theory of TCM and does not consider other influential factors such as the compatibility dosage of prescription and processed products, so studies on the mechanism of action of SGMD to restore symptoms of COVID-19 patients still needs to be further improved. 


\section{Declarations}

Ethics approval and consent to participate: Not applicable.

Consent for publication: I understand that the text and any pictures or videos published in the article will be freely available on the internet and may be seen by the general public. The pictures, videos and text may also appear on other websites or in print, may be translated into other languages or used for commercial purposes.

Availability of data and materials: All data generated or analysed during this study are included in this published article.

Competing interests: The authors declare no conflict of interest.

Funding: This research was funded by the Research Foundation of Administration of Traditional Chinese Medicine of Hebei Province (No. 2018105).

Author Contributions: Conceptualization: J. Li and D. Ma; methodology: Y. Jia and H. Sun; investigation: M. Si, H. Sun, and Y. Jia; writing-original draft preparation: H. Du; writing-review and editing: $\mathrm{H}$. Du and J. Li. All authors have read and agreed to the published version of the manuscript.

Acknowledgements: We should thank Yuguang Zheng هHebei Chemical \& Pharmaceutical College) for helpful discussions and excellent technical assistance.

\section{Abbreviations}

SGMD: Six Gentlemen Modified Decoction

COVID-19: Coronavirus Disease

TCM: Traditional Chinese Medicine

SGD: Six Gentlemen Decoction

\section{References}

1. Special Expert Group for Control of the Epidemic of Novel Coronavirus Pneumonia Preventive of the Chinese Medicine Association. An update on the epidemiological characteristics of novel coronavirus pneumonia(COVID-19). Chinese Journal of Viral Diseases.1-7.

2. National Health Commission and National Administration of Traditional Chinese Medicine. Diagnosis and treatment protocol for COVID-19 trial version 7. Jiangsu Journal of Traditional Chinese Medicine.2020;04:1-6. 
3. Zhang X. Effect of Xiangsha Liujunzi Decoction on active gastric ulcer and its recurrence. Guide of China Medicine.2019;03:184-185.

4. Yu XM, Liu F, and Zhang HY. Effect of Xiangsha Liujunzi Decoction on active gastric ulcer and its effect on recurrence. Chinese and Foreign Medical Research.2019;31:8-10.

5. Zhou TH. Observation on the therapeutic effect of xiangsha liujunzi decoction on active gastric ulcer. Nei Mongol Journal of Traditional Chinese Medicine.2019;10:35-36.

6. Xia BL. To explore the therapeutic effect of Xiangsha Liujunzi Decoction on chronic gastritis patients. Contemporary Medical Symposium.2019;15:202-203.

7. Tian FN, Ke J, Chen J, Lin HJ, Yang L, Zheng M, Liu YJ, Zeng XX, Zhou YN, and Yang Y. Investigation and analysis of TCM symptoms during recovery period in patients with COVID-19. Herald of Medicine.1-9.

8. Zong Y, Ding ML, Jia KK, Ma ST, and Ju WZ. Exploring active compounds of Da-Yuan-Yin in treatment of COVID-19 based on network pharmacology and molecular docking method. Chinese Traditional and Herbal Drugs.1-9.

9. Huang BS, Wang X, Li QQ, and Wang TL. Formononetin reduces inflammation in focal brain ischemia reperfusion ratvia inhibiting sphingosine kinase 1/sphingosine 1-phosphate signaling pathway. Acta Universitatis Medicinalis Nanjing(Natural Science).2017;10:1239-1242+1256.

10. Yuan CW, Xiong YY, Zhang QL, Liu XJ, Wei XD, and Song YP. Effects of formononetin on proliferation,apoptosis and miR-21/PTEN/AKT signaling pathway of HL-60 cells. Journal of Modern Oncology.2020;07:1062-1066.

11. Yu X, Fu BZ, Guo SZ, and Wang W. Protective effects research of kaempferol and formononetin on $\mathrm{H} 9 \mathrm{C} 2$ cells under hypoxi/reaeration condition by reducing reactive oxygen species. Liaoning Journal of Traditional Chinese Medicine.2017;01:111-113+222.

12. Wei $P$, Che ZB, Wang CE, Li DZ, and Chen KQ. Protective function and mechanism of quercetin in chronic obstructive pulmonary disease. Chinese Journal of Immunology.2019;21:2570-2575.

13. Venkateswara RC and Madhavan V. Effect of quercetin, flavonoids and alpha-tocopherol, an antioxidant vitamin, on experimental reflux oesophagitis in rats. European journal of pharmacology.2008;1-3.

14. Jeong $\mathrm{CE}$, Chee $\mathrm{KM}$, and Lee BH. Anti- and prooxidant effects of chronic quercetin administration in rats. European journal of pharmacology.2003;1-3.

15. Szeto YT and Benzie IFF. Effects of dietary antioxidants on human DNA exvivo. Free radical research.2002;1.

16. Akira M, Hitoshi A, and Junji T. Multitargeted cancer prevention by quercetin. Cancer letters.2008;2.

17. Zhai YY, Zhou L, and Lai YH. Research of quercetin on Antitumour activit. Journal of Modern Clinical Medical Bioengineering.2005;01:18-20.

18. Liu RY and Wang JD. Effects of Quercetin on human laryngeal cancer Hep-2 cells Journal of Clinical Otorhinolaryngology Head and Neck Surgery.2008;04:169-171. 
19. Zhong L, Chen FY, Wang HR, Wang C, and Ouyang RR. Effects of quercetin on cell morphology and expression of VEGF in K562 cell. Journal of Leukemia \& Lymphoma 2006;03:161-163.

20. Lee S, Lee HH, Su SY, Kang H, and Cho H. The anti-HSV-1 effect of quercetin is dependent on the suppression of TLR-3 in Raw 264.7 cells. Archives of pharmacal research.2017;5.

21. Marisa G, Celeste R, Saveria GMM, Laura C, Marina V, Roberta S, Roberta G, Gabriella D, Alberto F, and Mara C. Quercetin induces apoptosis and autophagy in primary effusion lymphoma cells by inhibiting PI3K/AKT/mTOR and STAT3 signaling pathways. The Journal of nutritional biochemistry. 2017.

22. Yao CG, Hu KH, Gao W, Cai XF, Qin JL, Lv SY, Du CH, and Wei YH. Inhibition of enterovirus 71 replication and viral 3C protease by quercetin. Virology journal.2018;1.

23. Chen C, Jiang ZY, Yu B, Wu XL, Dai CQ, Zhao CL, Ju DH, and Chen XY. Study on the anti-H1N1 virus effects of quercetin and oseltamivir and their mechanism related to TLR7 pathway. Journal of Asian Natural Products Research.2012;9.

24. Tian RX, Sun YZ, Yao YH, Zhang ZJ, Zhang JM, and Song WF. Effect of quercetin on immune function in immunocompromised mice. Modern Medicine Journal of China.2019;09:13-16.

25. Jiang R, Kao SJ, Xu XJ, and Xu DW. Effect of Quercetin on Humoral Immunity Function in Immunosuppressive Mice. Journal of Qingdao Agricultural University(Natural Science).2011;02:118$120+125$.

26. Wang JH, Zhang CN, Zhang JL, Xie J, Yang L, Xing YF, and Li ZF. The effects of quercetin on immunity, antioxidant indices, and disease resistance in zebrafish (Danio rerio). Fish physiology and biochemistry.2020;2.

27. Zhang GZ, Studies on metabolism of irisolidone in vovo and in votro of rats. 2014, Shenyang Pharmaceutical University.

28. Huang J, Zhu LM, and Zhu YQ. The relationship between KLF2/RelA imbalance and neutrophil apoptosis in asthmatic patients. Chinese Journal of Respiratory and Critical Care Medicine.2018;01:1-5.

29. Li M. Clinical significance of changes of serum TNF-a and IL-6 levels in eldery patients with chronic bronchial asthma. Journal of Radioimmunology.2005;06:440-441.

30. Umberto MG. Clinical review: a paradigm shift: the bidirectional effect of inflammation on bacterial growth. Clinical implications for patients with acute respiratory distress syndrome. Critical care (London, England).2002;1.

31. Bauer TT, Montón C, Torres A, Cabello H, Fillela X, Maldonado A, Nicolás JM, and Zavala E. Comparison of systemic cytokine levels in patients with acute respiratory distress syndrome, severe pneumonia, and controls. Thorax.2000;1.

32. Le HL and Luo GQ. Research progress of early posttraumatic inflammatory cytokines TNF-aIIL-1IL6. Modern Diagnosis and Treatment.2014;04:763-765.

33. Zhang G, Wang W, Li S, Yang H, Zhang M, Zhang P, Wen Y, Wu A, Yang L, Zhou B, and Chen X. IL6 gene allele-specific $\mathrm{C} / \mathrm{EBPa}$-binding activity affects the development of HBV infection through 
modulation of Th17/Treg balance. Genes and immunity.2015;8.

34. Mathew M, Milan F, Larry M, Najib A, James S, Liu GH, Avi S, Chan D, Otoniel MM, Madhuri C, and Antonio LC. Tocilizumab attenuates inflammation in ALS patients through inhibition of IL6 receptor signaling. American journal of neurodegenerative disease.2012;3.

35. Yang CM, Yang ZX, and Ma XL. The mechanism of AGEs-RAGE signaling pathway in diabetic nephropathy and the progress of Chinese Medicine treatment. Acta Chinese Medicine.2019;09:18641868.

36. Mélissa N and Pierre M. Selected cytokine pathways in rheumatoid arthritis. Seminars in immunopathology.2017;4.

37. Lu CH, Hsiu CO, Cecilia HD, Chen HI, Pai PY, Lee CY, Chen RJ, Chang RL, Viswanadha VP, Hsieh DJY, and Huang CY. Deep sea minerals ameliorate diabetic-induced inflammation via inhibition of TNFa signaling pathways. Environmental Toxicology.2020;4.

\section{Figures}

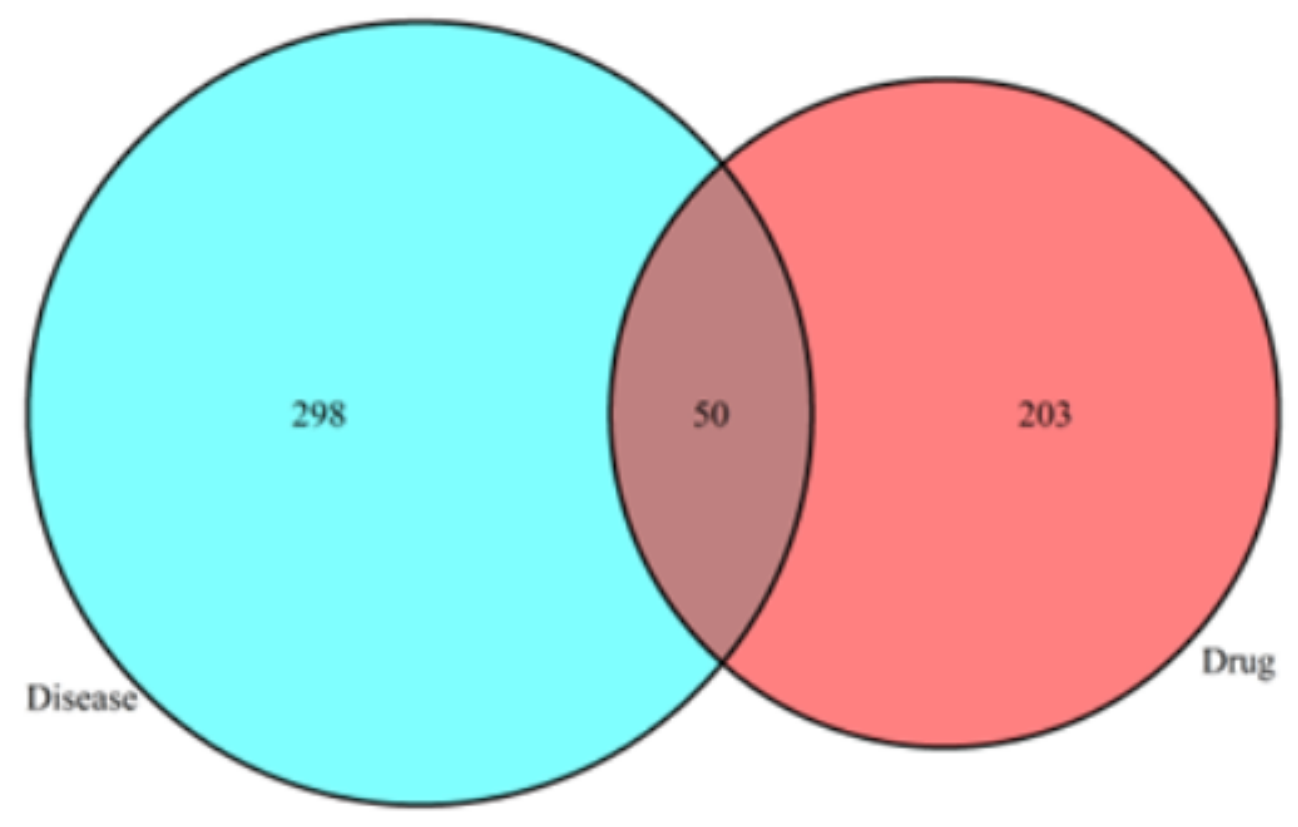

\section{Figure 1}

Venn diagram of SGMD and COVID-19 intersection targets. 


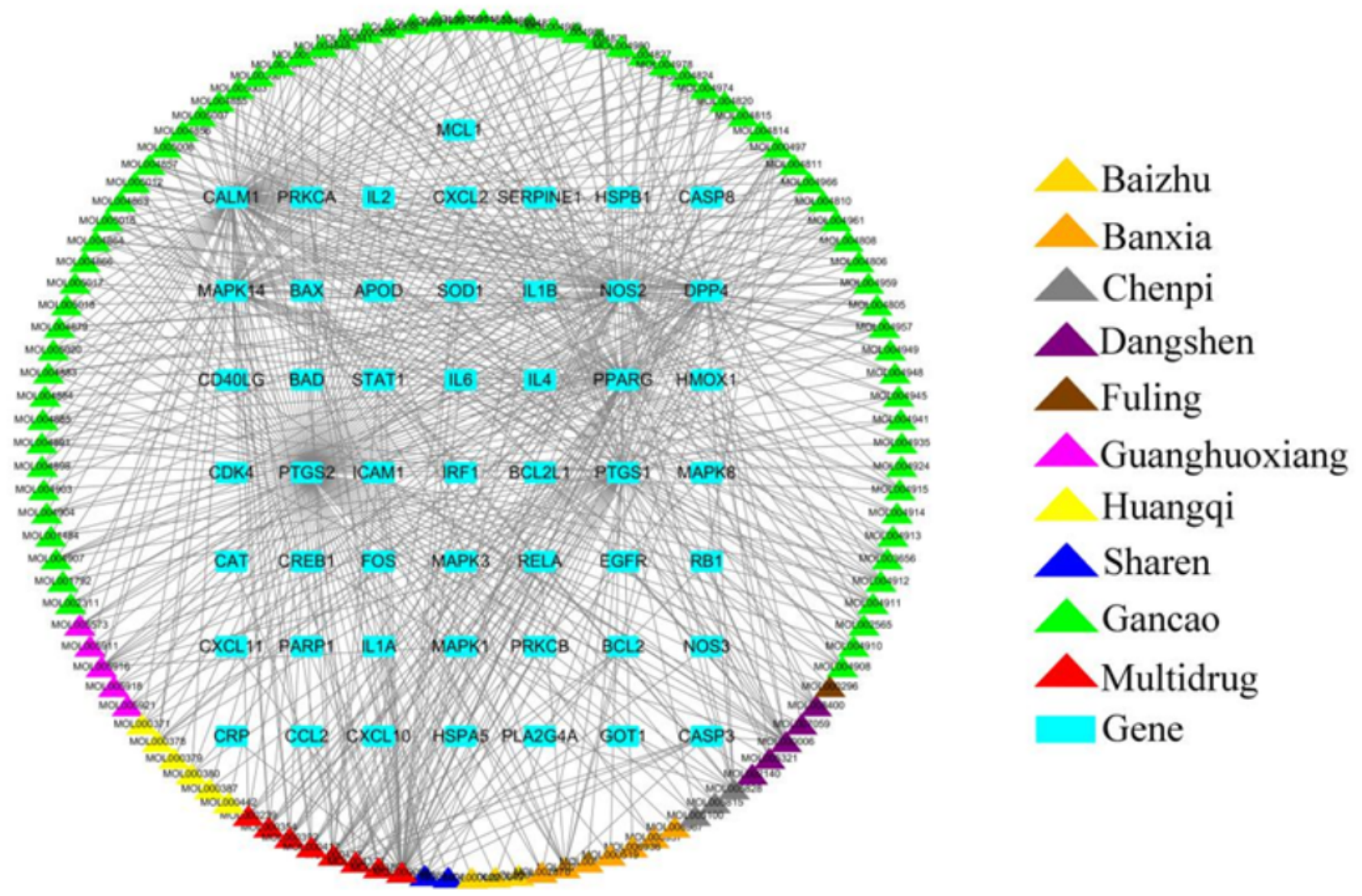

Figure 2

TCM compound regulatory network. The triangle represents the chemical composition, the square represents the target, and the side represents the relationship between the two 


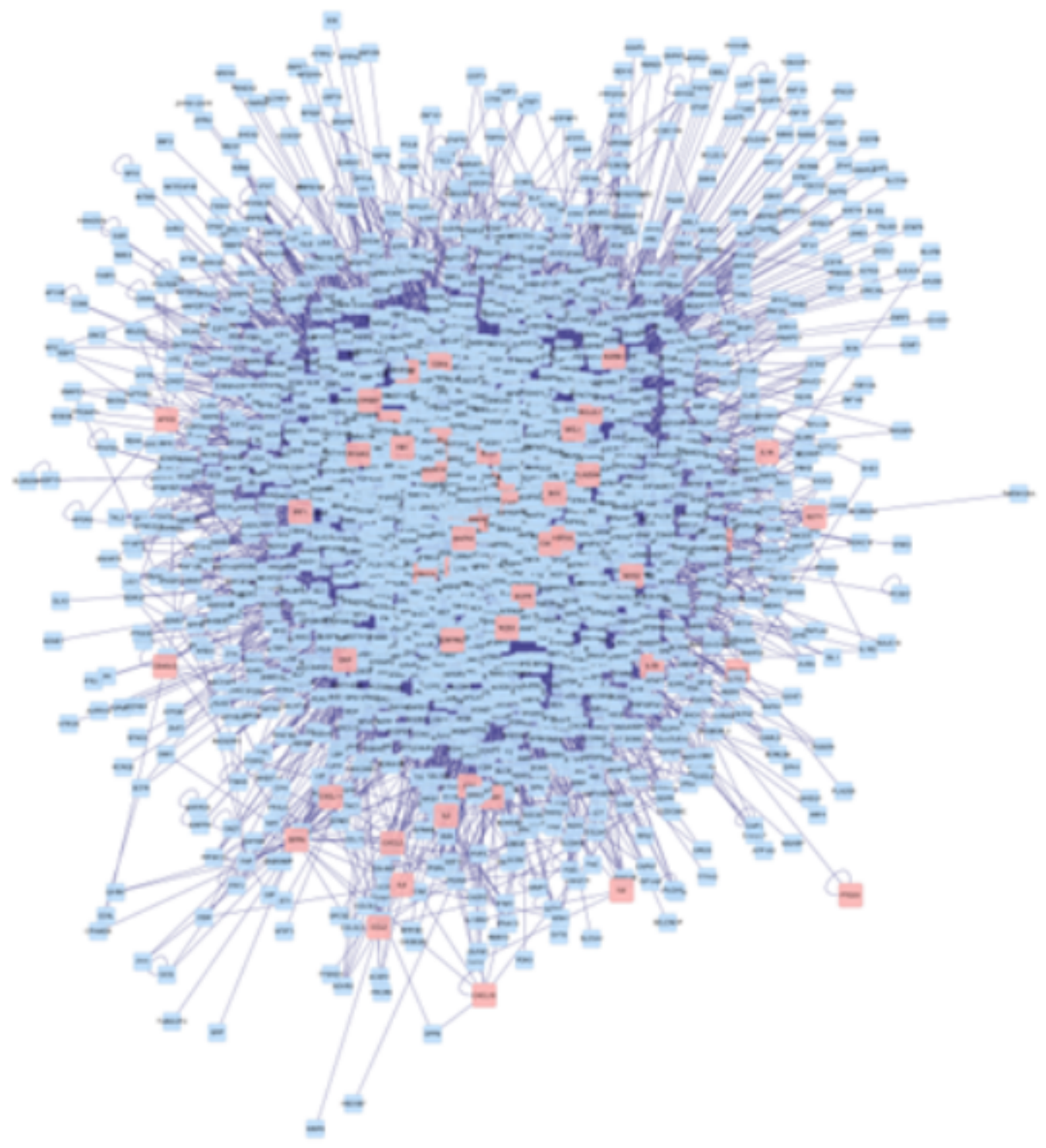

\section{Figure 3}

Protein-protein interaction network.

a

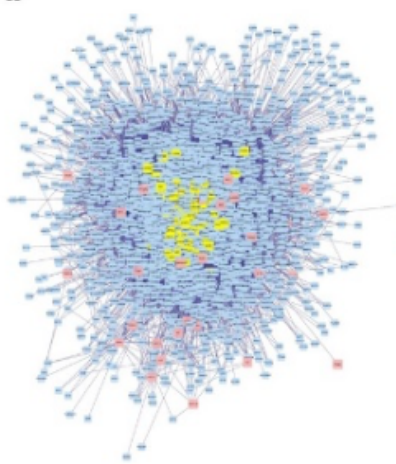

b

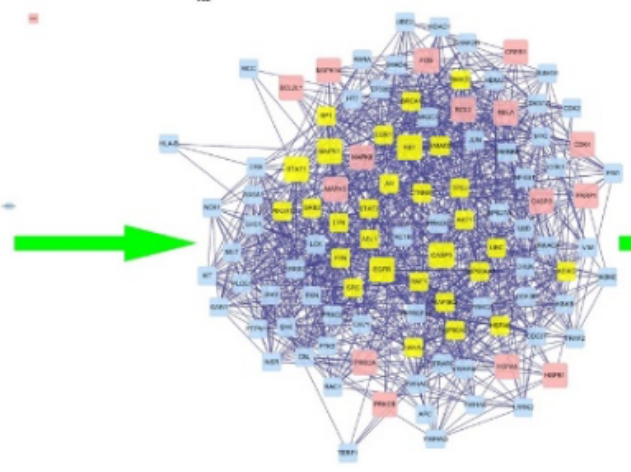

c

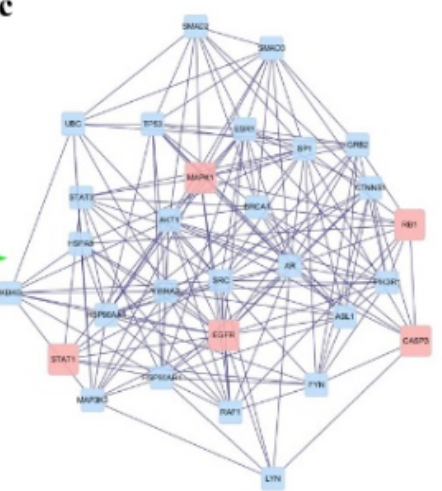


Figure 4

The PPI topology analysis
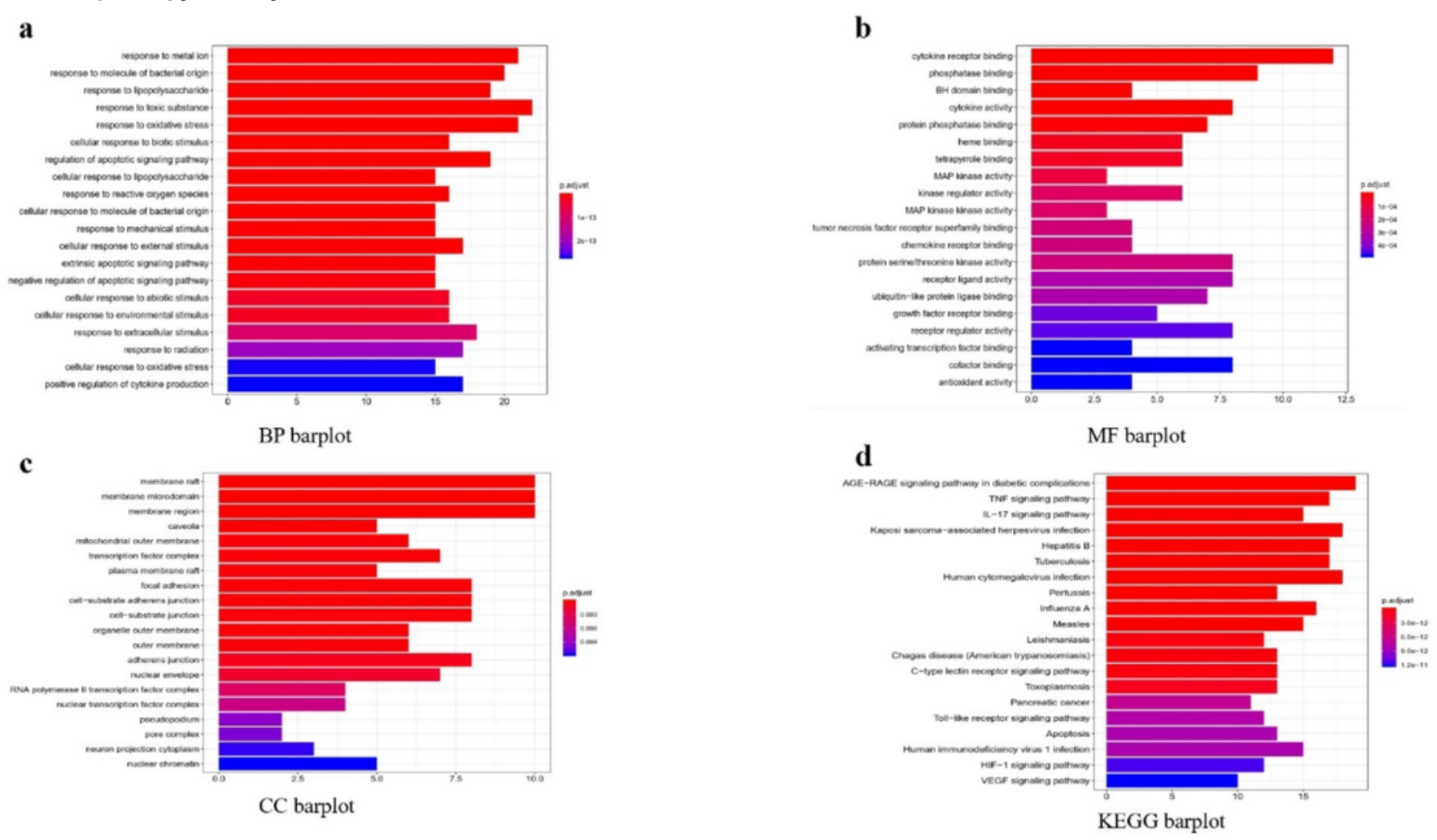

Figure 5

Analysis of the GO function ( $a, b$ and $c$ ) and the KEGG pathway (d) The abscissa represents the number of enrichments, the ordinate represents the name, and the color represents the significance. Redder the column corresponds to more significant in the enrichment. 


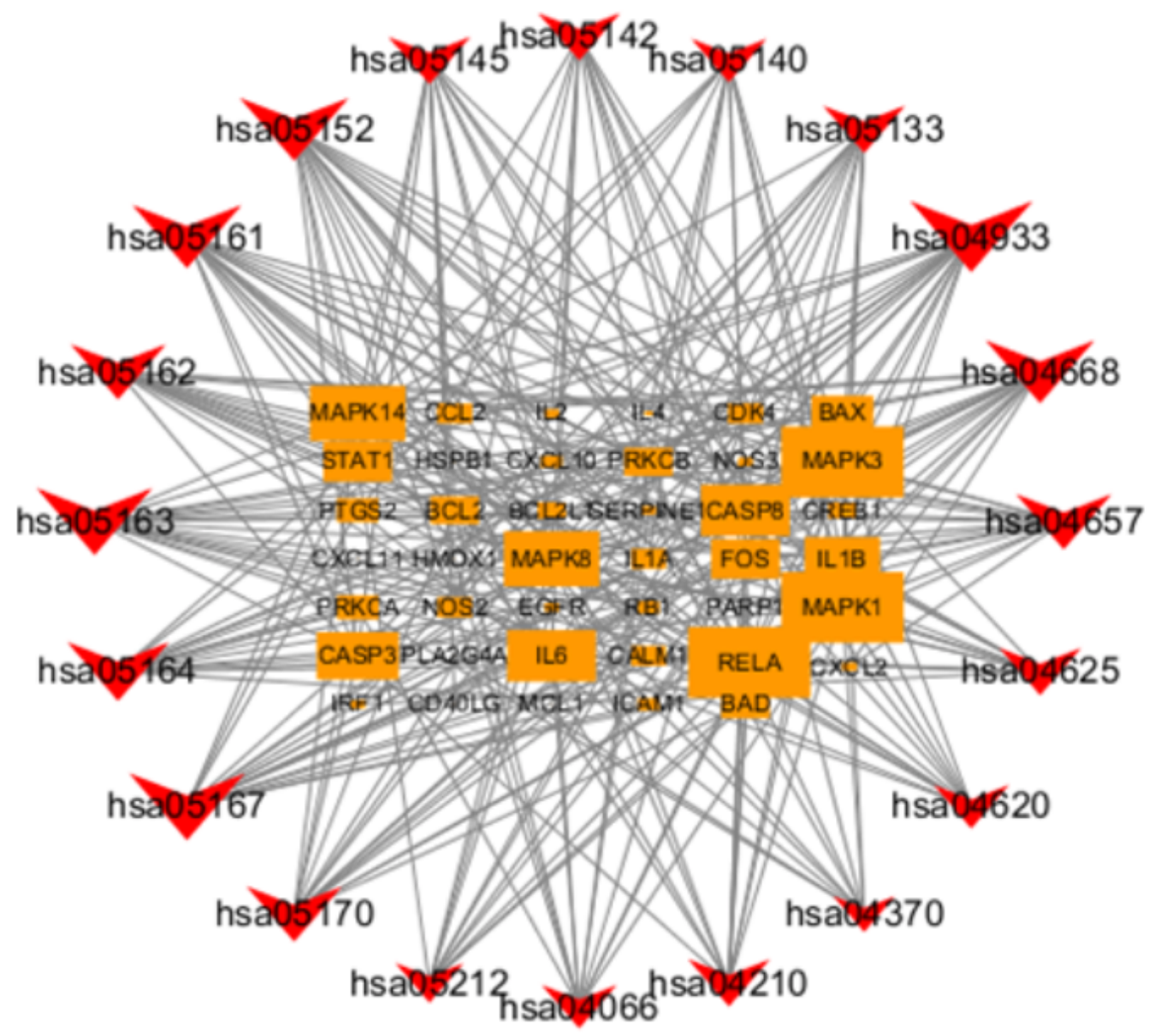

\section{Figure 6}

Target-pathway network. The yellow square nodes represent the targets, and the red v-shaped nodes represent the pathways. Larger squares correspond to more important targets in the pathway. 


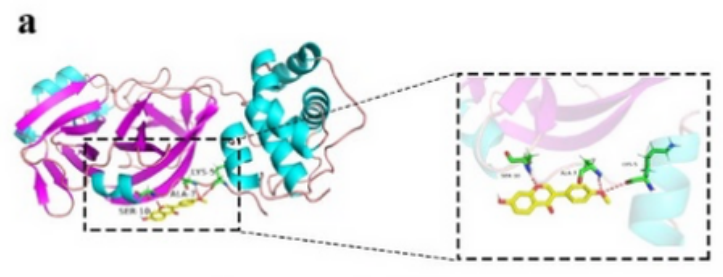

Formononetin-6lu7

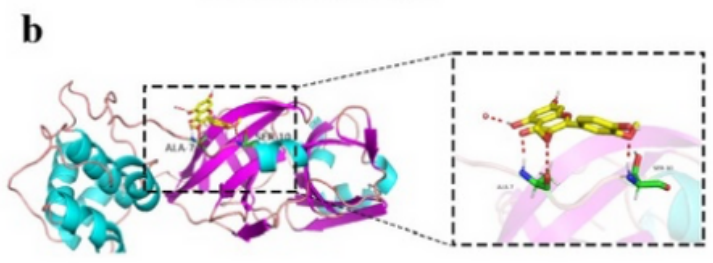

Quercetin der-6lu7

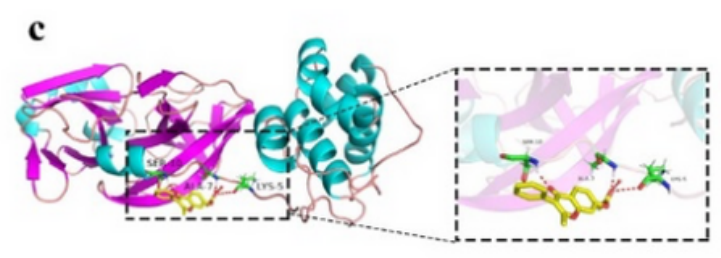

7-Acetoxy-2-methylisoflavone-6lu7

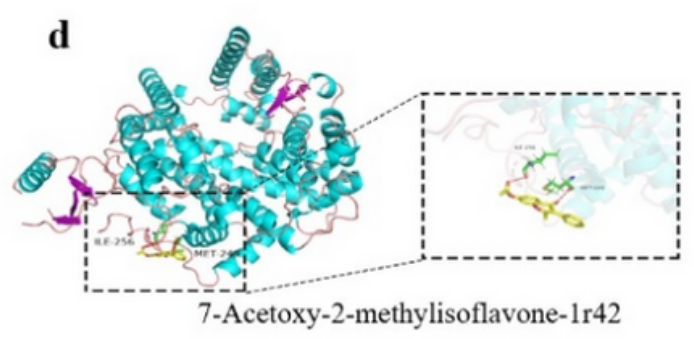

e

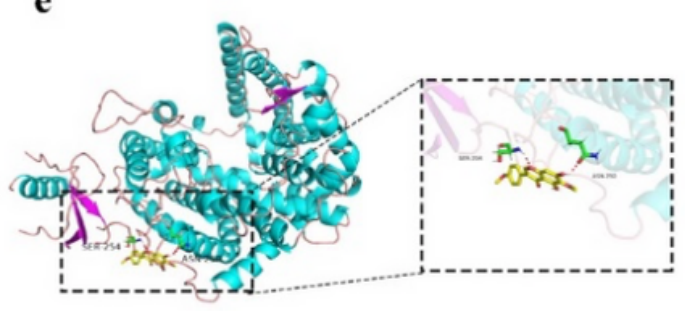

Iirisolidone-1r42

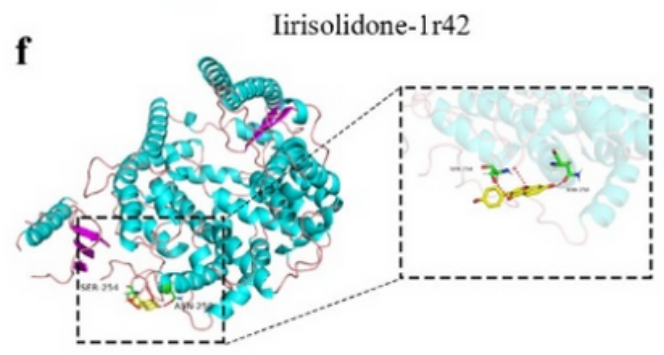

Formononetin-1r42

\section{Figure 7}

Results of docking models of 3 major effective ingredients docking with 2 proteins in the Systems Dock. The docking modes of formononetin, Quercetin der., 7-acetoxy-2-methylisoflavone and 6lu7 are on the left. The docking modes of 7-acetoxy-2-methylisoflavone, irisolidone, formononetin and 1r42 are on the right.

\section{Supplementary Files}

This is a list of supplementary files associated with this preprint. Click to download.

- GraphicAbstract.png

- GraphicAbstract.png 